\title{
Effect of freeze drying and hot air drying methods on quality of cordycepin production
}

\author{
Thitiphan Chimsook ${ }^{1, *}$ \\ ${ }^{1}$ Faculty of Science, Department of Chemistry and Applied Chemistry, Maejo University, Chiang Mai, Thailand
}

\begin{abstract}
This study determined the effect of drying methods including hot air drying and freeze drying on the quality of cordycepin production from Cordyceps militaris. The fruiting bodies and mycelium of $C$. militaris were used as the raw material. For hot air drying was performed at $55{ }^{\circ} \mathrm{C}$ for $24 \mathrm{hrs}$. Whereas the freeze drying was carried out under vacuum at $140 \mathrm{~L} \mathrm{~min}^{-1}$ for 48 hours. The bioactive compound extracted from dried powder of $C$. militaris from two drying methods was investigated. The results showed that both cordycepin and adenosine extracted from freezed drying sample had higher value than those of extracted from hot air dried sample. The bioactivities of $C$. militaris extract were investigated. The results revealed that the antioxidant activity and also total phenolic contents of $C$. militaris extract prepared from freeze drying had higher value than that of extracted from hot air drying. However, most of $C$. militaris production performed using hot air drying to dry sample because of its low cost technique.
\end{abstract}

Keywords: C. militaris, Cordycepin, Hot air drying, Freeze drying

\section{Introduction}

Drying of food is the method to remove the water in food. Drying is the application of warmth under controlled conditions to evaporation the water in liquid foods and produce the solid substance [1]. Because of the different products which have their own properties, there are developed different ways of drying. In general, the drying technologies compose of four principles including air drying, vacuum drying or puffing, spray drying and freeze drying, respectively. In this work, we evaluate two drying technologies of freeze drying and air drying (hot air drying) on quality of cordycepin production from Cordyceps militaris. Freeze-drying can preserve food and herb by extend and shelf life. With this technique it makes the material more convenient for transport. Freeze-drying is suitable for heat sensitive as it uses low processing temperature. The procedure of freeze-drying starts from freezing the material, then reducing the pressure and adding heat to allow the frozen water in the material to sublimate [2-4]. Compared to conventional food preservation technologies, the key benefits of freeze-drying include the following: retains original characteristics of the product such as colour, taste and size, reconstitutes to original state when placed in water, shelf stable at room temperature, reduces the weight of product, offers highest quality in a dry product compared to other drying methods $[5,6]$. For hot air drying was invented to improve the efficient for drying herb and plant samples. The design of hot air drying was presented in Fig. 1 which showed the inside and outside figures of hot air drying.

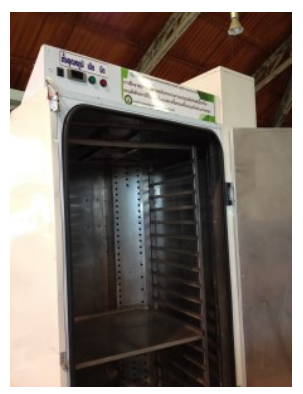

Fig. 1. The design of hot air drying machine [7].

This invention was designed to save time and cost in drying process. From Fig. 1, the inside of oven composed of many shelves for the trays. For the operation, the automatic program was setted up [7]. This hot air drying machine can be enhanced the dehydration of various plants and also C. militaris. C. militaris is one of the most important traditional Chinese medicines. It has studied its pharmacological properties. The pharmacological properties are attributed to various active substances such as $\beta$-glucans, cordycepin, adenosine etc. [8,9]. In this work, cordycepin (3'deoxyadenosine) was extracted from $C$. militaris. Cordycepin, a nucleoside analog, was first isolated from C.militaris. It has been regarded as a medicinal agent

\footnotetext{
* Corresponding author: thitiphan.cs@gmail.com
} 
responsible for immunological regulation [10-12], anticancer [13], antifungus [14], antivirus [15], antihyperlipidemia [15] and antileukemia [16-18] activities. The quantity and quality of cordycepin production involved with the culture techniques and also drying methods. Therefore, this study chiefly highlights the drying methods on quality of cordycepin production from C. militaris.

\section{Material and methods}

\subsection{Drying methods}

Fresh of fruiting bodies and mycelium of C. militaris were a kind gift from SM Trading and Service Col, ltd. The factory was located in Maejo, Chiang Mai, Thailand. For hot air drying, the hygienic dryer was designed related to thermal engineer and food safety. All components of the dryer were suitable temperature distribution and accelerated moisture transfer out of system as shown in Fig. $1.50 \mathrm{~g}$ of the fruiting bodies and mycelium of $C$. militaris were used as the material sample and dried at $55^{\circ} \mathrm{C}$ for $24 \mathrm{hrs}$. For the freeze drying process, $50 \mathrm{~g}$ of $C$. militaris was frozen at $-40{ }^{\circ} \mathrm{C}$ and freeze dried under vacuum at $140 \mathrm{~L} \mathrm{~min}^{-1}$ for 48 hours. The grinding machine was used to perform the powder freeze dried sample. All dried samples were prepared the cordycepin extract to investigate the quality of dried C. militaris sample after drying process.

\subsection{Determinations of total phenolic content}

The fruiting bodies and mycelium from freeze drying and hot air drying were dried to constant weight and homogenized to powder. About $20 \mathrm{~g}$ of dried sample in $500 \mathrm{ml}$ ethanol was extracted by ultrasonic cell crushing, and then centrifuged at $10000 \mathrm{~g}$ for $15 \mathrm{~min}$. The residue was re-extracted and then combined the supernatant before evaporated on a water bath at $55{ }^{\circ} \mathrm{C}$ until dry. The total phenolic content of extract was determined with Folin-Ciocalteu reagent according to the method of Khan et al. and Naczk $M$ et al. [19, 20]. A $1.0 \mathrm{~mL}$ aliquot of sample was added to $1.5 \mathrm{~mL}$ deionized water and $0.5 \mathrm{~mL}$ of $0.1 \mathrm{~mol} / \mathrm{L}$. Folin-Ciocalteu reagent. The contents were mixed thoroughly immediately and added $1.0 \mathrm{~mL}$ of $20 \%$ sodium carbonate solution then mixed thoroughly. The controls contained all the reaction reagents except the sample. After $30 \mathrm{~min}$, the sample was incubated at $37{ }^{\circ} \mathrm{C}$ and then measured the absorbance at $750 \mathrm{~nm}$. The results were compared to a gallic acid calibration curve. The total phenolic content was expressed as $\mathrm{mg}$ gallic acid equivalent (GAE) $/ \mathrm{kg}$ of dry weight (DW).

\subsection{Determinations of antioxidant activities}

The antioxidant activities of all substances were determined by DPPH assay. The methods was modified from that of Kim DO. et al. and Gulcin I. et al. [21, 22]. DPPH assay was based on the reduction of DPPH radical solution in the presence of hydrogen donating antioxidants. The DPPH radical solution $(0.8 \mathrm{mM})$ in $95 \%$ ethanol was prepared. The extract $(500 \mu \mathrm{l})$ was diluted to $5.0 \mathrm{ml}$ using deionized water and $95 \%$ ethanol $(1: 1 \mathrm{~V} / \mathrm{V})$ and then $0.5 \mathrm{ml} \mathrm{DPPH}$ solution was added. The mixed solution was shaken vigorously. The sample was determined the absorbance after mixing. Trolox was used as chemical standard. The results were expressed in mg Trolox equivalents/kg of dry weight (DW). The assay was performed in triplicate.

\subsection{Extraction of cordycepin and adenosine}

The extraction of cordycepin and adenosine from dried C. militaris obtained from two drying methods was carried out prior to analysis the quantity of them by HPLC. All dried samples were extracted the bioactive compounds including cordycepin and adenosine. Dried samples were grinded into powder. Then, approximate $1.0 \mathrm{~g}$ of sample was precisely weighed and added into 10 $\mathrm{ml} 95 \%$ ethanol and deionized water $(1: 1 \mathrm{~V} / \mathrm{V})$ in a 50 $\mathrm{ml}$ centrifuge tube. The mixed sample was subsequently placed in an ultrasonic machine for extracting cordycepin and adenosine at a power of 75 watt. After the centrifugation, the sample extraction procedure was repeated another twice. Supernatant obtained from the three times centrifugation was mixed and exactly measured of its volume. The sample was filtrated through a $0.45 \mu \mathrm{m}$ filter prior to HPLC analysis [23].

\subsection{Determination of the cordycepin and adenosine}

All HPLC analysis work was carried out on Agillent model 1100 series, Agillent, Germany. Standards of cordycepin and adenosine were purchased from SigmaAldrich. The standard adenosine and cordycepin solvent was consecutively injected five times to draw calibration curves. The determination condition of the sample was set by using the ratio of mobile phase of water and methanol at $85: 15(\mathrm{~V} / \mathrm{V}), 90: 10(\mathrm{~V} / \mathrm{V})$ and $92: 8(\mathrm{~V} / \mathrm{V})$, respectively. The separation was conducted in isocratic elution with a flow rate of $1.0 \mathrm{ml} / \mathrm{min}$. The detection wavelength of photo-diode array was set at $210-400 \mathrm{~nm}$ and the column temperature was $30{ }^{\circ} \mathrm{C}$. The injection volume was $10 \mu \mathrm{l}$ [23].

\subsection{Determinations of morphology of dried C. militaris powder}

The morphology of dried C. militaris powder from two drying methods was investigated by Scanning Electron Microscopy (JSM 5410-LV, JEOL, Japan) with the tungsten filament $\mathrm{K}$ type, accelerate voltage of $10.0 \mathrm{kV}$ and working distance of $15 \mathrm{~mm}$. The $C$. militaris powder was milled sieved. Gold coating was performed by finer coater (JFC 1200, JEOL, Japan) for $150 \mathrm{sec}$.

\section{Results and discussion}

Cordycepin is one of the most important biologically active metabolites. In this work, cordycepin was extracted from dried powder of $C$. militaris. In general, drying of plant, food and active ingredients may be performed in different dryers and methods according to the initial properties, final desired characteristic of the products and economic factors. In this work, two drying 
methods of freeze drying and hot air drying were chosen to prepare the dried $C$. militaris before extracted the active compounds. The quantity and bioactivities of extracted active compounds may be revealed the efficiency of drying techniques to keep the precious substances from dried $C$. militaris. In recent years, researchers made remarkable progress in cordycepin production. They focused their attention on the three aspects to improve cordycepin production including strain improving, optimizing ingredients of medium and optimizing culture conditions, respectively, However, the drying process played an important role in the preservation of precious nutrients from $C$. militaris. Nowadays, the most drying method used to dry the fresh fruiting bodies and mycelium of $C$. militaris is hot air drying before grinded to fine powder. The hot air drying is chosen to dry the fruiting bodies and mycelium of $C$. militaris because it is the low cost technique, faster and provided higher yield than freeze drying. However, this study was conducted in order to evaluate the effect of different drying methods on quality of cordycepin production from $C$. militaris. The drying methods were the hot air drying at $55{ }^{\circ} \mathrm{C}$ for 24 hours and freeze drying for 48 hours. The active compound, bioactivities and also morphology of C. militaris extract from different drying methods were determined to clearify that drying methods had effected to the quality of cordycepin production. The results shown in Table 1 were the average yields, productivity and moisture contents of $C$. militaris powder obtained from hot air drying and freeze drying.

Table 1. Parameters of $C$. militaris powder obtained from the hot air drying and freeze drying.

\begin{tabular}{|l|c|c|}
\hline \multicolumn{1}{|c|}{ Parameters } & $\begin{array}{c}\text { Hot air } \\
\text { drying }\end{array}$ & Freeze drying $^{\mathbf{1}}$ \\
\hline Yield (\%) & $65.00 \pm 1.42^{\mathrm{a}}$ & $57.6 \pm 1.78^{\mathrm{b}}$ \\
\hline Productivity $\left(\mathrm{g} \mathrm{h}^{-1}\right)$ & $1.35 \mathrm{a}$ & $0.6 \mathrm{~b}$ \\
\hline Moisture (\%) & $7.04 \pm 0.19^{\mathrm{a}}$ & $4.15 \pm 0.20^{\mathrm{b}}$ \\
\hline
\end{tabular}

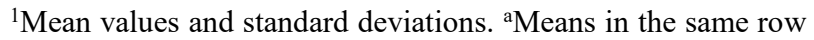
with different superscripts differ significantly $(\mathrm{p}<0.05)$ by the t-test.

The drying method performed the different yields of C. militaris powder. The higher yield of grinded powder after drying was obtained from hot air drying at $65.00 \%$ whereas lower yield at $57.6 \%$ was found from the freeze drying. On the other hand, the moisture contents of $C$. militaris powder were $7.04 \%$ and $4.15 \%$ for hot air drying and freeze drying, respectively. These results revealed that the freeze drying can improve the efficient of dehydration process. The dehydration process can decrease the moisture contents of samples and it is very important on quality of $C$. militaris powder. The dried samples from two drying methods were prepared the $C$. militaris extracts by using ultrasonic cell crushing with $95 \%$ ethanol : deionized water at $1: 1(\mathrm{~V} / \mathrm{V})$ as solvent in order to evaluate the bioactivities. including total phenolic contents and antioxidant activities using DPPH assay. Moreover, the bioactive substances of C. militaris including cordycepin and adenosine were carried out after extracting with $95 \%$ ethanol : deionized water at $1: 1(\mathrm{~V} / \mathrm{V})$ and determined by HPLC analysis. All results were shown in Table 2. The antioxidant activity and also total phenolic contents of $C$. militaris extract from the freeze drying method were higher than those of the hot air drying method. In addition, both cordycepin and adenosine extracted from freezed drying sample had high value when compared to that from hot air drying sample.

Table 2. Bioactivities and bioactive compounds of $C$. militaris powder obtained from the hot air drying and freeze drying.

\begin{tabular}{|l|c|c|}
\hline \multicolumn{1}{|c|}{ Parameters } & Hot air drying & Freeze drying \\
\hline $\begin{array}{l}\text { Total phenolic } \\
\text { content (mg } \\
\text { GAE/kg (DW)) }\end{array}$ & $3,197.50^{\mathrm{a}}$ & $3,597.20^{\mathrm{b}}$ \\
\hline $\begin{array}{l}\mathrm{mg} \text { Trolox } \\
\text { equivalents/kg } \\
\text { sample (DW) }\end{array}$ & $24,012.15^{\mathrm{a}}$ & $28,816.00^{\mathrm{b}}$ \\
\hline $\begin{array}{l}\text { Cordycepin } \\
\text { (mg/kg DW) }\end{array}$ & $1950.50^{\mathrm{a}}$ & $2,317.10^{\mathrm{b}}$ \\
\hline $\begin{array}{l}\text { Adenosine } \\
\text { (mg/kg DW) }\end{array}$ & $989.35^{\mathrm{a}}$ & $1,097.05^{\mathrm{b}}$ \\
\hline
\end{tabular}

${ }^{a}$ Means in the same row with different superscripts differ significantly $(\mathrm{p}<0.05)$ by the $\mathrm{t}$-test.

The results revealed that different drying methods had significant effects on quantity and quality of cordycepin production from C. militaris. A literature search showed that drying methods had a significant effect on physical and chemical properties and also bioactive compounds. It can be concluded that the drying methods had effected on the proportion of the bioactive components in substances. Similar results of bioactive compounds were found in C. militaris and also C. sinensis which prepared with freeze drying methods by L. Huang et al. [23]. However, spray drying method was one of the best drying method which can help to remove the moisture in the sample. Due to its versatility and speed, spray drying became the most used drying technique for heat sensible substances such as foods and biological materials. For $C$. militaris, the spray drying method may not preserve the nutrient and bioactive compounds.

The surface morphology and size of $C$. militaris powder were analysed by SEM. All SEM images of $C$. militaris powder from the freeze drying and hot air drying methods were shown in Fig. 2a and Fig 2b, respectively. From Fig. 2a and Fig $2 b$, it can be seen in SEM images that $C$. militaris powder from both drying methods was similar which appeared as irregular shapes with variable sizes. However, the previous work have reported that the pure cordycepin crystals were in the form of rod-like crystals with a smooth surface [24]. For this work, it can be concluded that the drying methods have not affected the morphology of C. militaris powder. 


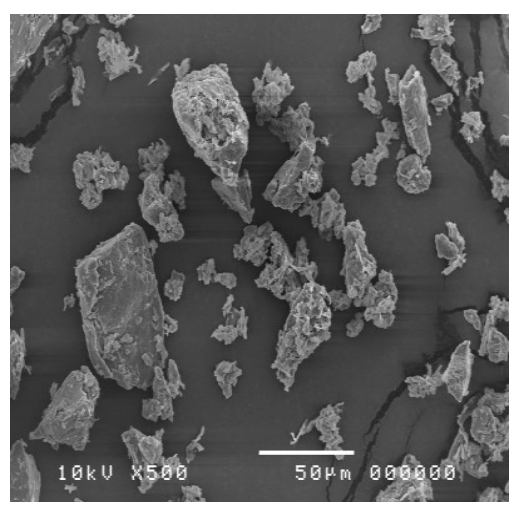

(a)

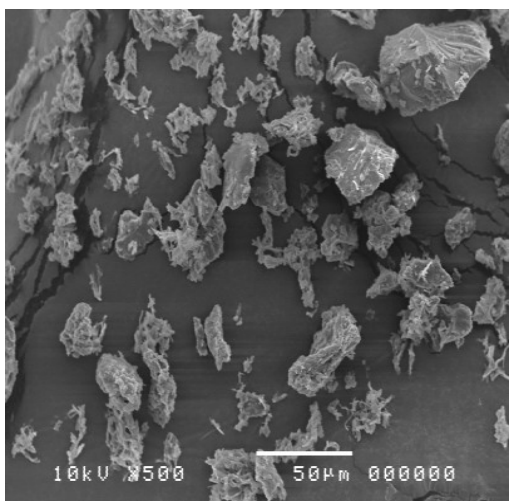

(b)

Fig. 2. SEM of $C$. militaris powder from (a) freeze drying method (b) hot air drying method.

\section{Conclusion}

Freeze drying and hot air drying methods have been successfully applied to dry $C$. militaris sample. All samples were extracted to evaluate the total phenolic contents and antioxidant activities. The bioactive compounds of $C$. militaris including cordycepin and adenosine were extracted and investigated. In term of the physical properties, hot air dried powders showed a promising result for easy handling of the dried sample when compared to the freeze drying process. The cost of hot air drying process was cheaper than that of freeze drying. However, the antioxidant activities and total phenolic contents of dried samples from freeze drying had higher value than that of hot air drying. Moreover, the cordycepin and adenosine which be extracted from freeze drying samples were higher than that of be extracted from hot air drying samples. The morphology of both drying methods was similar. All morphology of samples showed the irregular shapes with variable sizes and wrinkled shapes which were the result of low density particles. The XRD pattern and also morphology of cordycepin which extracted from each dried samples will be studied in further work.

\section{Acknowledgement}

This work was supported by NNR-National Science and Technology Development Agency and Faculty of Science,
Department of Chemistry, Applied Chemistry, Maejo University, Thailand.

\section{References}

1. E.F.do E. Santo, L.K.F.de Lima, A.P.C. Torres, G.de Oliveiras and E.H.G. Ponsano, Food. Sci. Technol. 33, 1 (2013)

2. A. Ciurzynska and A. Lenart, Pol. J. Food Nutr. Sci 61 (2011)

3. F. Franks, Eur. J. Pharm. Biopharm. 45 (1998)

4. F. Franks, Cryobiology. 40 (2000)

5. J. Gawalek, Inż. Roln. 71 (2005)

6. J.P. George and A.K. Datta, J. Food. Eng. 52 (2002)

7. T. Chimsook and R. Assawarachan, Key. Eng. Mater. 735 (2017)

8. Q. Zhang, Y. Liu, Z. Di, C.C. Han and Z. Liu, Fungal. Genom. Biol. 6, 1 (2016)

9. S.K. Das, M. Masuda, A. Sakurai and M. Sakakibara, Fitoterapia. 81, 8 (2010)

10. D.D. De Silva, S. Rapior, F. Fons, A.H. Bahkali and K.D. Hyde, Fungal. Divers. 55 (2012)

11. X. Zhou, C.U. Meyer, P. Schmidtke and F. Zepp, Eur. J. Pharmacol. 453 (2002)

12. E.M. Noh, J.S. Kim, H. Hur, B.H. Park and E.K. Song, Rheumatology. 48 (2009)

13. N. Yoshikawa, S. Yamada and C. Takeuchi, Naunyn. Schmiedebergs. Arch. Pharmacol. 77 (2008)

14. A.M. Sugar and R.P. McCaffrey, Antimicrob. Agents. Chemother. 42 (1998)

15. D.D. De Silva, S. Rapior, K.D. Hyde and H. Bahkali, Fungal. Divers. 56 (2012)

16. D.D. De Silva, S. Rapior and E. Sudarman, Fungal. Divers. 62 (2013)

17. E.N. Kodama, R.P. McCaffrey, K. Yusa and H. Mitsuya, Biochem. Pharmacol. 59 (2000)

18. H. Thomadaki, C.M. Tsiapalis and A. Scorilas, Cancer. Chemother. Pharmacol. 61 (2008)

19. R.A. Khan, M.R. Khan, S. Sahreen and M. Ahmed, Chem. Central. J. 6 (2012)

20. M. Naczk and F. Shahidi, J. Chromatogr. A. 1054 (2004)

21. D.O. Kim, S.W. Jeong and C.Y. Lee, Food. Chem. 81 (2003)

22. I. Gulcin, Arch. Toxicol. 86 (2012)

23. L. Huang, Q. Li, Y. Chen, X. Wang and X. Zhou, Afr. J. Microbiol. Res. 3, 12 (2009)

24. J.-Q. Zhang, D. Wu, K.-M. Jiang, D. Zhang, X. Zheng, C.-P. Wan, H.-Y. Zhu, X.-G. Xie, Y. Jin, J. Lin, Carbohydr. Res. 406 (2015) 